\title{
Basicity of Systems of Sines with Linear Phase in Weighted Sobolev Spaces
}

\author{
V. F. Salmanov ${ }^{1}$ and A. R. Safarova ${ }^{2}$ \\ ${ }^{1}$ Institute of Mathematics and Mechanics of NAS of Azerbaijan, B. Vahabzade 9, \\ 1141 Baku, Azerbaijan \\ ${ }^{2}$ Nakhchivan State University, University Campus, 7012 Nakhchivan City, Azerbaijan \\ Correspondence should be addressed to V. F. Salmanov; valid.salmanov@mail.ru
}

Received 6 February 2013; Revised 24 April 2013; Accepted 24 April 2013

Academic Editor: Giuseppe Carbone

Copyright (c) 2013 V. F. Salmanov and A. R. Safarova. This is an open access article distributed under the Creative Commons Attribution License, which permits unrestricted use, distribution, and reproduction in any medium, provided the original work is properly cited.

The perturbed systems of sines, which appear when solving some partial differential equations by the Fourier method, are considered in this paper. Basis properties of these systems in weighted Sobolev spaces of functions are studied.

\section{Introduction}

When solving many problems in mathematical physics by Fourier method (see e.g., [1-4]), there appear perturbed systems of sines and cosines of the following form:

$$
\begin{gathered}
\{\sin (n t+\alpha(t))\}_{n=1}^{\infty}, \\
1 \cup\{\cos (n t+\alpha(t))\}_{n=1}^{\infty},
\end{gathered}
$$

where $\alpha(t)=\beta t+\gamma, \beta, \gamma \in R$ are real parameters. Using Fourier method requires the study of basis properties of the above systems in Lebesgue and Sobolev spaces of functions. Relevant investigations date back to the well-known works by Paley and Wiener [5] and Levinson [6]. For $\gamma=0$, basis properties of these systems in spaces $L_{p}, 1<p<+\infty$, are completely studied in [7-12]. The case of weighted $L_{p}$ was considered by E. I. Moiseev in [13, 14]. Basis properties of some perturbed systems of exponents in Sobolev spaces are studied in [15-19]. Further references include [20-23].

Our paper is devoted to the study of basis properties of these systems in weighted Sobolev spaces. Unlike previous works, we offer a different method of investigation.

\section{Auxiliary Facts}

Let $L_{p, \rho}(0, \pi)$ and $W_{p, \rho}^{1}(0, \pi)$ be weighted Lebesgue and Sobolev spaces with the following norms:

$$
\|u\|_{L_{p, \rho}}^{p}=\int_{0}^{\pi}|u(\theta)|^{p} \rho(\theta) d \theta, \quad\|u\|_{W_{p, \rho}^{1}}=\|u\|_{L_{p, \rho}}+\left\|u^{\prime}\right\|_{L_{p, \rho}},
$$

where $\rho(\theta)=\theta^{a}(\pi-\theta)^{b}\left|\theta-\theta_{0}\right|^{c}, \theta_{0} \in(0, \pi), a, b, c \in$ $(-1,+\infty)$. Denote by $\mathscr{L}_{p, \rho}$ the following direct sum:

$$
\mathscr{L}_{p, \rho}=L_{p, \rho} \oplus C,
$$

where $C$ is a complex plane. The norm in this space is defined as follows: $\|\widehat{u}\|_{\mathscr{L}_{p, \rho}}=\|u\|_{L_{p, \rho}}+|\lambda|$, where $\widehat{u}=(u, \lambda) \in \mathscr{L}_{p, \rho}$. The following easily provable lemmas play an important role in obtaining our main result.

Lemma 1. Let $p \in(1,+\infty), a, b, c \in(-1, p-1)$. Then the operator

$$
A(u ; \lambda)=\lambda+\int_{0}^{t} u(\theta) d \theta
$$

performs an isomorphism between the spaces $\mathscr{L}_{p, \rho}$ and $W_{p, \rho}^{1}(0, \pi)$; that is, the spaces $\mathscr{L}_{p, \rho}$ and $W_{p, \rho}^{1}$ are isomorphic. 
Proof. First we show the boundedness of this operator. We have the following:

$$
\begin{aligned}
\|A \widehat{u}\|_{W_{p, \rho}} & \left\|\lambda+\int_{0}^{t} u(\theta) d \theta\right\|_{L_{p, \rho}}+\|u\|_{L_{p, \rho}} \\
= & \|\lambda\|_{L_{p, \rho}}+\left\|\int_{0}^{t} u(\theta) d \theta\right\|_{L_{p, \rho}}+\|u\|_{L_{p, \rho}} \\
= & \left(\int_{0}^{\pi}|\lambda|^{p} \rho(\theta) d \theta\right)^{1 / p}+\left(\int_{0}^{\pi}\left|\int_{0}^{t} u(\theta) d \theta\right|^{p} \rho(\tau) d \tau\right)^{1 / p} \\
& +\|u\|_{L_{p, \rho}} \\
\leq & |\lambda|\|\rho\|_{L_{1}}^{1 / p}+\left(\int_{0}^{\pi}\left(\int_{0}^{t}|u(\theta)| d \theta\right)^{p} \rho(\tau) d \tau\right)^{1 / p}+\|u\|_{L_{p, \rho}} \\
\leq & |\lambda|\|\rho\|_{L_{1}}^{1 / p}+\left(\int_{0}^{\pi}\left(\int_{0}^{\pi}|u(\theta)| d \theta\right)^{p} \rho(\tau) d \tau\right)^{1 / p} \\
& +\|u\|_{L_{p, \rho}} \\
= & |\lambda|\|\rho\|_{L_{1}}^{1 / p}+\int_{0}^{\pi}|u(\theta)| d \theta\|\rho\|_{L_{1}}^{1 / p}+\|u\|_{L_{p, \rho} \cdot}
\end{aligned}
$$

Applying Hlder's inequality, we obtain the following:

$$
\begin{aligned}
\int_{0}^{\pi}|u(\theta)| & d \theta \\
& =\int_{0}^{\pi}|u(\theta)| \rho^{1 / p} \rho^{-(1 / p)} \\
& \leq \int_{0}^{\pi}|u(\theta)|^{p} \rho d \theta\left(\int_{0}^{\pi} \rho^{-(q / p)}(\theta) d \theta\right)^{1 / q} \\
& =\|u\|_{L_{p, \rho}}\left\|\rho^{1 /(1-p)}\right\|_{L_{1}}^{1 / q}, \quad \text { where } \frac{1}{p}+\frac{1}{q}=1
\end{aligned}
$$

Consequently,

$$
\begin{aligned}
\|A \widehat{u}\|_{W_{p, \rho}} & \leq|\lambda|\|\rho\|_{L_{1}}^{1 / p}+\|u\|_{L_{p, \rho}}\|\rho\|_{L_{1}}^{1 / p}\left\|\rho^{1 /(1-p)}\right\|_{L_{1}}^{1 / q}+\|u\|_{L_{p, \rho}} \\
& =|\lambda| \rho_{L_{1}}^{1 / p}+\left(\|\rho\|_{L_{1}}^{1 / p}\left\|\rho^{1 /(1-p)}\right\|_{L_{1}}^{1 / q}+1\right)\|u\|_{L_{p, \rho}} \\
& \leq M\left(|\lambda|+\|u\|_{L_{p, \rho}}\right)=M\|\widehat{u}\|_{L_{p, \rho}}
\end{aligned}
$$

where $M=\max \left\{\|\rho\|_{L_{1}}^{1 / p} ;\|\rho\|_{L_{1}}^{1 / p}\left\|\rho^{1 /(1-p)}\right\|_{L_{1}}^{1 / q}\right\}$.

Let us show that $\operatorname{Ker} A=\{0\}$. Let $A \widehat{u}=0$; that is,

$$
\lambda+\int_{0}^{t} u(\theta) d \theta=0, \quad \forall t \in(0, \pi),
$$

where $\lambda \in C, u \in L_{p, \rho}$. Differentiating this equation, we obtain $u(\theta)=0$ a.e. on $(0, \pi)$.

It follows that $\lambda=0$. From (9) it directly follows that $u=0$ a.e. on $(0, \pi)$ and this implies that $\widehat{u}=0$.

Show that $\operatorname{Im} A=W_{p, \rho}^{1}(0, \pi)$. Let $v \in W_{p, \rho}^{1}(0, \pi)$ be an arbitrary function. Assume $\widehat{v}=\left(v^{\prime}, v(0)\right)$. It is clear that $A \widehat{v}=$ $v$ and $\widehat{v} \in L_{p, \rho}$. Then by Banach theorem we find that the operator $A$ has a bounded inverse. This proves Lemma 1.

Now let us prove the following lemma.

Lemma 2. Let $p \in(1,+\infty)$ and $a, b, c \in(-1, p-1)$. Then for all $p_{0} \in(1, \alpha): L_{p, \rho} \subset L_{p_{0}}(0, \pi)$, where $\alpha=\min \{p ; p /(a+$ $1) ; p /(b+1) ; p /(c+1) ; 2\}$.

Proof. Let $f \in L_{p, \rho}(0, \pi), p_{0} \in(1, \alpha)$. We have the following:

$$
\begin{array}{rl}
\int_{0}^{\pi}|f|^{p_{0}} & d t \\
& =\int_{0}^{\pi}|f|^{p_{0}} \rho^{p_{0} / p} \rho^{-\left(p_{0} / p\right)} d t \\
& \leq\left(\int_{0}^{\pi}|f|^{p} \rho d t\right)^{p_{0} / p}\left(\int_{0}^{\pi} \rho^{p_{0} /\left(p_{0}-p\right)} d t\right)^{\left(p-p_{0}\right) / p} \\
& =\|f\|_{L_{p, \rho}^{p_{0}}}\left(\int_{0}^{\pi} t^{a p_{0} /\left(p_{0}-p\right)}\left|t-t_{0}\right|^{c p_{0} /\left(p_{0}-p\right)}\right.
\end{array}
$$

$$
\left.(\pi-t)^{b p_{0} /\left(p_{0}-p\right)} d t\right)^{\left(p-p_{0}\right) / p}
$$

As $p_{0}<p /(a+1)$ and $a>-1$, then $a p_{0} /\left(p_{0}-p\right)>-1$. Similarly, we find that $b p_{0} /\left(p_{0}-p\right)>-1$ and $c p_{0} /\left(p_{0}-p\right)>$ -1 . It is easy to see that $f \in L_{p_{0}}(0, \pi)$ and, moreover,

$$
\|f\|_{p_{0}} \leq C\|f\|_{L_{p, \rho}} .
$$

This proves the lemma.

From results of the paper [24] it follows the validity of the following lemma.

Lemma 3. Let $f \in L_{p, \rho}(0, \pi), \rho=t^{a}\left|t-t_{0}\right|^{c}(\pi-t)^{b}, a, b, c \in$ $(-1, p-1)$, and $f(t)=\sum_{n=1}^{\infty} c_{n} \sin (n+\beta / 2) t$ in $L_{p, \rho}(0, \pi)$. Then the series $\sum_{n=1}^{\infty}\left(c_{n} /(n+\beta / 2)\right)$ converges absolutely.

\section{Main Result}

Theorem 4. Let $p \in(1,+\infty), a, b, c \in(-1, p-1)$. Then system (2) forms a basis for $W_{p, \rho}^{1}(0, \pi)$ if and only if system (1) forms a basis for $L_{p, \rho}(0, \pi)$, where $\alpha(t)=(\beta / 2) t, \quad \beta \neq-2 k, k=$ $1,2,3, \ldots$.

Proof. First let us assume that the system

$$
\left\{\sin \left(n+\frac{\beta}{2}\right) t\right\}_{n=1}^{\infty}
$$

forms a basis for $L_{p, \rho}(0, \pi)$. Show that the system $\left\{\widehat{u}_{n}(t)\right\}_{n=0}^{\infty}$ forms a basis for $\mathscr{L}_{p, \rho}$, where $\widehat{u}_{0}=(0 ; 1), \widehat{u}_{n}=(-(n+$ 
$\beta / 2) \sin [(n+\beta / 2) t] ; 1)$, for $n \geq 1$. It suffices to show that every element $\widehat{u}=(u ; \lambda)$ of space $\mathscr{L}_{p, \rho}$ can be expanded in a unique way as a series of the following:

$$
\widehat{u}=\sum_{n=0}^{\infty} \lambda_{n} \widehat{u}_{n}
$$

that is,

$$
\begin{gathered}
u(t)=-\sum_{n=1}^{\infty}\left(n+\frac{\beta}{2}\right) \lambda_{n} \sin \left[\left(n+\frac{\beta}{2}\right) t\right] \\
\lambda=\lambda_{0}+\sum_{n=1}^{\infty} \lambda_{n} .
\end{gathered}
$$

Since system (1) forms a basis for $L_{p, \rho}(0, \pi)$, we have expansion (14), and the coefficients $\left\{\lambda_{n}\right\}_{n=1}^{\infty}$ are determined uniquely. By Lemma 3 , the series $\sum_{n=1}^{\infty} \lambda_{n}$ converges absolutely. Then, it is clear that the number $\lambda_{0}$ in (15) is determined uniquely. And this implies that the system $\left\{\widehat{u}_{n}\right\}_{n=0}^{\infty}$ forms a basis for $\mathscr{L}_{p, \rho}$.

Consider the system $\left\{v_{n}\right\}_{n=0}^{\infty}$, where $v_{n}=A \widehat{u}_{n}, n \geq 0$, $A \widehat{u}=A[(u ; \lambda)]=\lambda+\int_{0}^{t} u(\tau) d \tau$, and $\widehat{u}=(u ; \lambda) \in \mathscr{L}_{p, \rho}$. By Lemma 1 , the operator $A$ is an isomorphism in $L\left(W_{p, \rho}^{1} ; \mathscr{L}_{p, \rho}\right)$. Then the system $\left\{v_{n}\right\}_{n=0}^{\infty}$ forms a basis for $W_{p, \rho}^{1}(0, \pi)$. It is easy to see that

$$
v_{0}=1, \quad v_{n}=\cos \left(n+\frac{\beta}{2}\right) t, \quad n \geq 1 .
$$

Now we prove the converse. Assume that the system $1 \mathrm{U}$ $\{\cos (n+\beta / 2) t\}_{n=1}^{\infty}$ forms a basis for $W_{p, \rho}^{1}(0, \pi)$.

Consider the system $\widehat{u}_{n}=A^{-1} v_{n}, n \geq 0$. The inverse operator $A^{-1}$ is defined as $A^{-1} v=\left(\nu^{\prime} ; \nu(0)\right)$. It is absolutely clear that the system $\left\{\widehat{u}_{n}\right\}_{n=0}^{\infty}$ forms a basis for $\mathscr{L}_{p, \rho}$ and

$$
\widehat{u}_{0}=(0,1), \quad \widehat{u}_{n}=\left(-\left(n+\frac{\beta}{2}\right) \sin \left(n+\frac{\beta}{2}\right) t ; 1\right) .
$$

Consequently, $\widehat{u}=(u ; \lambda) \in \mathscr{L}_{p, \rho}$ has a unique expansion of the form (14), (15) in $\mathscr{L}_{p, \rho}$. As a result, we obtain that for all $u \in L_{p, \rho}$ has an expansion with respect to system (1) in $L_{p, \rho}$. Suppose there exists another expansion of $u$ in $L_{p, \rho}$ :

$$
u(t)=\sum_{n=1}^{\infty}\left(n+\frac{\beta}{2}\right) \mu_{n} \sin \left(n+\frac{\beta}{2}\right) t
$$

Then Lemma 3 implies the absolute convergence of series $\sum_{n=1}^{\infty} \mu_{n}$

Assume $\mu_{0}=\lambda-\sum_{n=1}^{\infty} \mu_{n}$. It is clear that the biorthogonal coefficients of the element $\widehat{u}=(u ; \lambda)$ are $\left(\bar{\mu} ; \mu_{0}\right)$, where $\bar{\mu}=$ $\left\{\mu_{n}\right\}_{n=1}^{\infty}$.

Then, from basicity of system $\left\{\widehat{u}_{n}\right\}_{n=0}^{\infty}$ in $\mathscr{L}_{p, \rho}$, we obtain that $\mu_{n}=\lambda_{n}$, for all $n \geq 0$. So we came upon a contradiction which proves the theorem.

\section{References}

[1] S. M. Ponomarev, "On an eigen value problem," Doklady Akademii Nauk SSSR, vol. 249, no. 5, pp. 1068-1070, 1979.

[2] E. I. Moiseev, "Some boundary value problems for mixed-type equations," Differential Equations, vol. 28, no. 1, pp. 105-115, 1992.

[3] E. I. Moiseev, "Solution of the Frankl problem in a special domain," Differentsial'nye Uravneniya, vol. 28, no. 4, pp. 721-723, 1992.

[4] E. I. Moiseev, "On existence and uniqueness of solution a classical problem," Doklady Rossiǔskaya Akademiya Nauk, vol. 336, no. 4, pp. 448-450, 1994.

[5] R. Paley and N. Wiener, Fourier Transforms in the Complex Domain, vol. 19 of American Mathematical Society Colloquium Publications, American Mathematical Society, Providence, RI, USA, 1934.

[6] N. Levinson, Gap and Density Theorems, vol. 29 of American Mathematical Society Colloquium Publications, American Mathematical Society, Providence, RI, USA, 1940.

[7] A. M. Sedletskiǔ, "Biorthogonal Expansions of functions in series of exponents on intervals of the Real Axis," Uspekhi Matematicheskikh Nauk, vol. 37, no. 5(227), pp. 51-95, 1982.

[8] E. I. Moiseev, "The basis property for systems of sines and cosines," Doklady Akademii Nauk SSSR, vol. 275, no. 4, pp. 794798, 1984.

[9] E. I. Moiseev, "On the basis property of a system of sines," Differentsial'nye Uravneniya, vol. 23, no. 1, pp. 177-179, 1987.

[10] B. T. Bilalov, "Basicity of some systems of exponents, cosines and sines," Differentsial'nye Uravneniya, vol. 26, no. 1, pp. 10-16, 1990.

[11] B. T. Bilalov, "Basis properties of some systems of exponents, cosines and sines," Sibirskii Matematicheskii Zhurnal, vol. 45, no. 2, pp. 264-273, 2004.

[12] S. R. Sadigova, "On one method for establishing a basis from double systems of exponentials," Applied Mathematics Letters, vol. 24, no. 12, pp. 1969-1972, 2011.

[13] E. I. Moiseev, "On basicity of systems of cosines and sines in weight space," Differentsialnye Uravneniya, vol. 34, no. 1, pp. 4044, 1998.

[14] E. I. Moiseev, "The basicity in the weight space of a system of eigen functions of a differential operator," Differentsial'nye Uravneniya, vol. 35, no. 2, pp. 200-205, 1999.

[15] E. I. Moiseev, "On differential properties of expansions in the system of sines and cosines," Differentsial'nye Uravneniya, vol. 32, no. 1, pp. 117-126, 1996.

[16] A. M. Sedletskiur, "Approximate properties of the systems of exponents in Sobolev spaces," Vestnik Moskovskogo Universiteta I, no. 4, pp. 3-8, 1999.

[17] Z. G. Huseynov and A. M. Shykhammedov, "On bases of sines and cosines in Sobolev spaces," Applied Mathematics Letters, vol. 25, no. 3, pp. 275-278, 2012.

[18] D. L. Russell, "On exponential bases for the Sobolev spaces over an interval," Journal of Mathematical Analysis and Applications, vol. 87, no. 2, pp. 528-550, 1982.

[19] V. F. Salmanov and V. S. Mirzoev, "On basicity of a system of functions in the space $W_{p}^{1}(0, \pi)$," Proceedings of Institute of Mathematics and Mechanics of the National Academy of Sciences of Azerbaijan, vol. 33, no. 41, pp. 153-156, 2010.

[20] X. He and H. Volkmer, "Riesz bases of solutions of SturmLiouville equations," The Journal of Fourier Analysis and Applications, vol. 7, no. 3, pp. 297-307, 2001. 
[21] B. T. Bilalov, "On basicity of systems of exponents, cosines and sines in $L_{p}$," Doklady Rossijskoj Akademii Nauk, vol. 365, no. 1 , pp. 7-8, 1999.

[22] B. T. Bilalov, "On basicity of some systems of exponents, cosines and sines," Doklady Rossijskoj Akademii Nauk, vol.379, no. 2, pp. 7-9, 2001.

[23] B. T. Bilalov, "Bases from the exponents, cosines and sines being the eigen values of differential operators," Differentsial'nye Uravneniya, vol. 39, no. 5, pp. 1-5, 2003.

[24] M. H. Karakash, "The Housdorff-Young and Paley type theorems for one system of sines," Proceedings of Institute of Mathematics and Mechanics of the National Academy of Sciences of Azerbaijan, vol. 23, no. 31, pp. 59-64, 2005. 


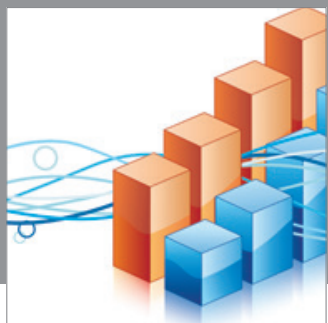

Advances in

Operations Research

mansans

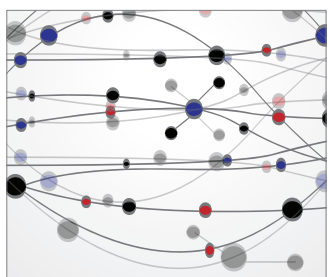

The Scientific World Journal
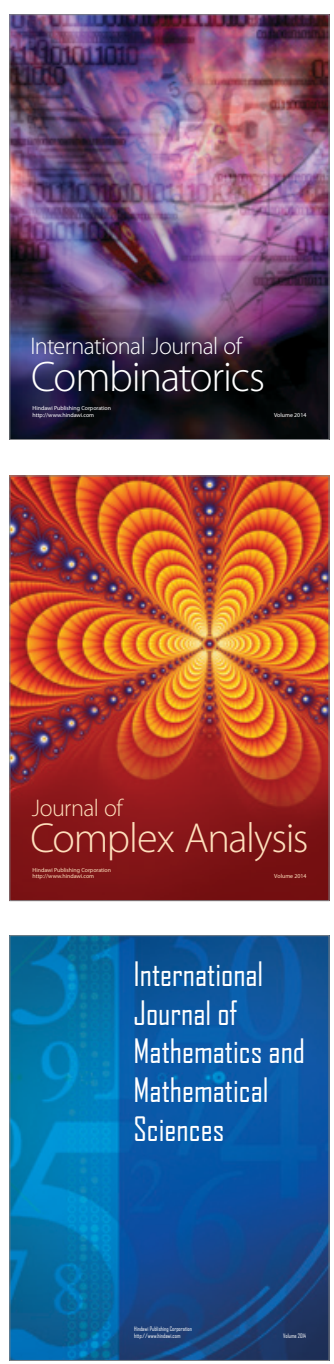
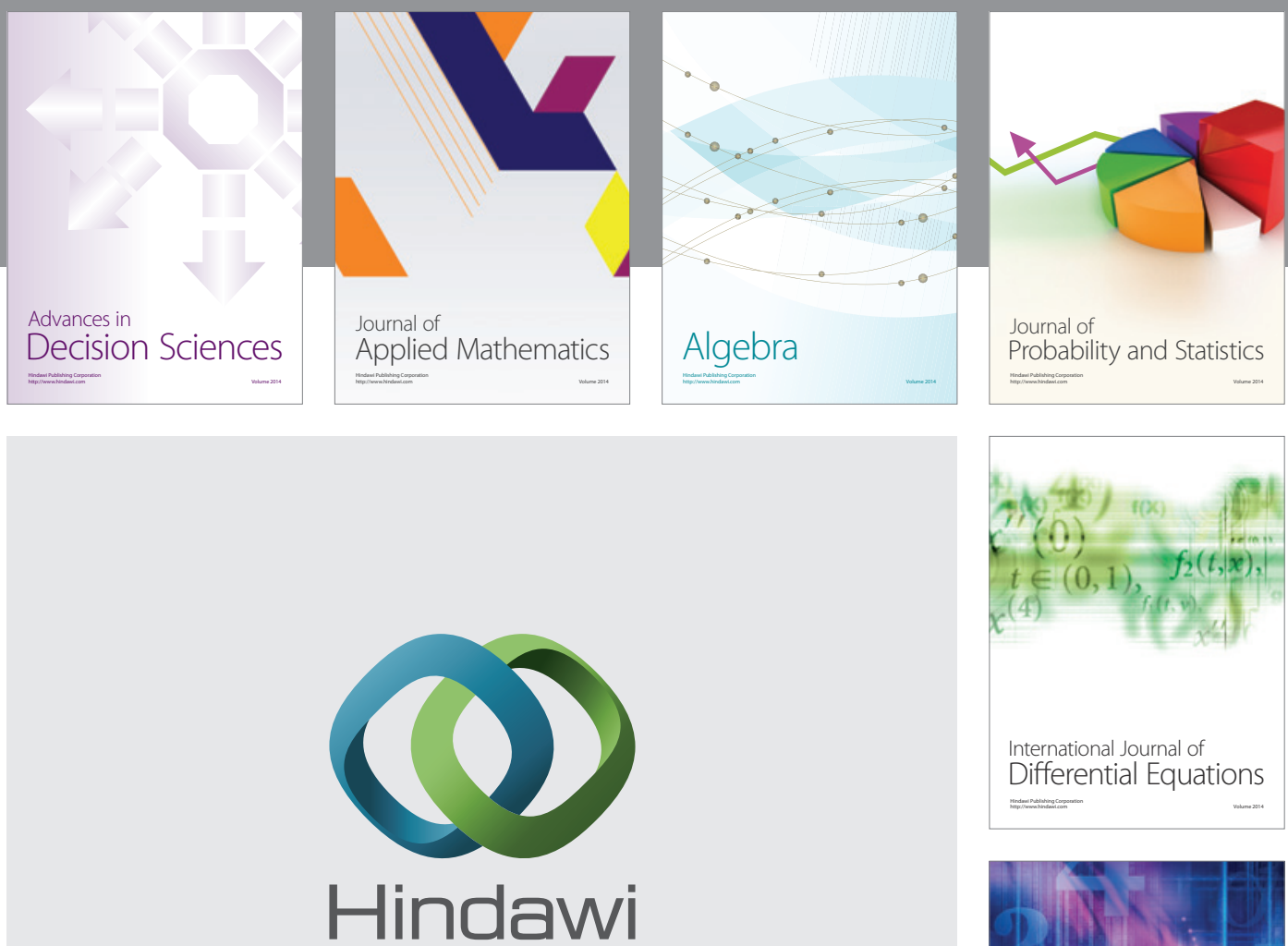

Submit your manuscripts at http://www.hindawi.com
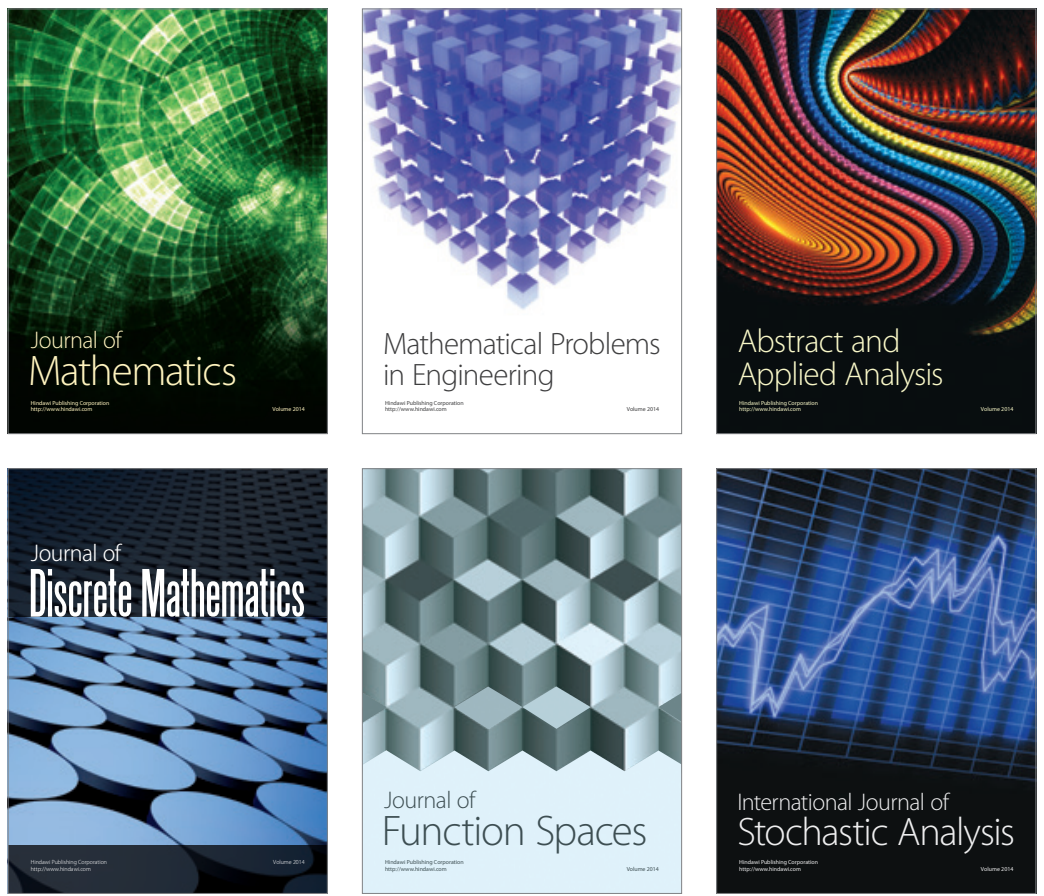

Journal of

Function Spaces

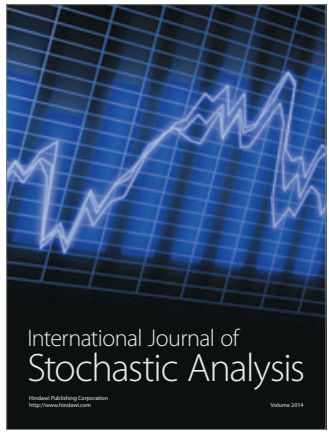

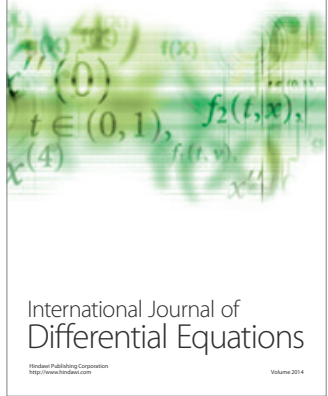
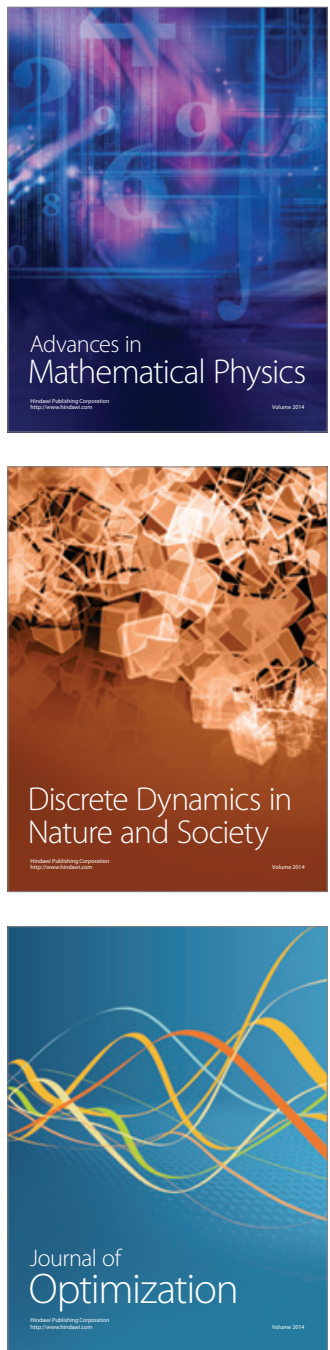